\title{
Sind Sie sicher?
}

IT-Sicherheit und Cybercrime sind keine neuen Themen. Spätestens seit nahezu alle mit dem Internet verbunden sind, sind sie allgegenwärtig, ob privat oder im beruflichen Umfeld. Durch die verstärkte Nutzung von Videokonferenzsystemen und anderen digitalen Werkzeugen aufgrund der Pandemie, sind sie aber erneut ins Zentrum der Aufmerksamkeit gerückt.

Der Mensch bleibt dabei die Hauptschwachstelle, gezielt werden Mitarbeiter angegriffen, um Zugriff auf Unternehmensdaten zu erlangen. Das sogenannte Phishing - also das Versenden von gefälschten E-Mails mit dem Zweck, an persönliche Informationen wie Passworte oder Kreditkartennummern zu gelangen - ist in letzter Zeit oft professioneller geworden und lässt sich deshalb nicht mehr so leicht erkennen wie in der Vergangenheit. Letztlich bleibt es immer beim alten Hase-Igel-Spiel zwischen Kriminellen und Verteidigern. Komplett verhindern lassen sich solche Attacken nicht, aber der angerichtete Schaden kann minimiert werden.

Aber nicht nur Privatpersonen und Unternehmen sind das Ziel von Cyberkriminellen. Gerade auch Staaten werden immer wieder das Ziel solcher Attacken. Meist wird dabei von der IT-Infrastruktur der jeweiligen Regierungen geredet, von Bundesbehörden etwa. Wenig im Blick ist dagegen, dass es insbesondere kleinere Kommunen sind, die nur unzureichend auf solche Angriffe vorbereitet sind. Ganz aktuell hat Bitterfeld darunter zu leiden gehabt. Im Juli führte eine Cyberattacke dort dazu, dass die Verwaltung für nahezu zwei Wochen weitgehend lahmgelegt wurde - der Katastrophenfall musste ausgerufen werden, wie Thomas Lang in seinem Kommentar in dieser Ausgabe beschreibt. Generell, so Lang, müssten kritische Infrastrukturen besser geschützt werden.

All diese Beispiele zeigen, dass das Erpressungspotenzial, das die umfassende Digitalisierung mit sich bringt, enorm ist. Alle Beteiligten sind gut beraten, sich auf den Fall der Fälle vorzubereiten. Ganz vermeiden lassen sich erfolgreiche Einbruchsversuche nicht, aber der mögliche Schaden kann erheblich und wirksam reduziert werden, wenn man entsprechende Maßnahmen ergreift.

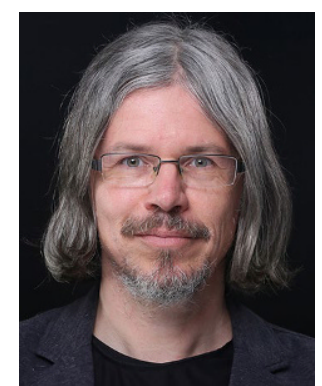

Peter Pagel $(\triangle)$

peter.pagel@springer.com

Wiesbaden, Deutschland

In diesem Sinne, bleiben Sie wachsam und helfen Sie Kollegen und Mitarbeitern dabei, es ebenfalls zu sein.

Herzliche Grüße

Peter Pagel

Chefredakteur

Wirtschaftsinformatik \& Management 2022 • 14 (1): 1 https://doi.org/10.1365/s35764-022-00385-8

Angenommen: 4. Januar 2022

○) The Author(s), under exclusive licence to Springer Fachmedien Wiesbaden GmbH, ein Teil von Springer Nature 2022 